\title{
Le nouveau procédé Biostyr : l'azote et le phosphore traités à grande vitesse
}

\author{
R. Rogalla*, C. Paffoni**, A. Vidal*, M. Badard ${ }^{* * *}$ \\ Anjou Recherche, Chemin de la Digue, 78600 Maisons-Laffitte \\ * Criter-SIAAP, 82 Av. Kléber, 92700 Colombes \\ *** OTV, 11 av. Dubonnet, 92407 Courbevoie
}

\section{Introduction}

Les nouvelles normes européennes imposent l'élimination de l'azote et du phosphore des rejets urbains en zones sensibles. En Europe, des accords sur la lutte contre la pollution de la mer du Nord et de la mer Baltique obligent à traiter à des niveaux avancés toutes les eaux usées des bassins versants des grands fleuves se déversant dans les mers intérieures, et notamment les effluents de toutes les villes côtières. En mer Méditerranée, ainsi que sur les côtes atlantiques, le développement croissant d'algues incite les autorités à réduire la concentration d'éléments eutrophisants tels que les sels nutritifs.

Les moyens classiques pour éliminer l'azote et le phosphore des eaux usées exigent un espace considérable et s'intègrent mal aux endroits à forte densité de population qui sont aussi les plus forts générateurs de rejets. Des procédés plus performants ont pour objectif de rapprocher les stations de traitement à la source en les rendant plus compactes, afin de réduire les coûts d'infrastructure; un mètre de canalisation coûte à peu près l'équivalent de l'épuration d'un habitant.

\section{Technologies à Grande Vitesse en Temps Record...}

Plusieurs nouvelles méthodes de traitement voient le jour chaque année au Centre de recherche de Maisons-Laffitte. Le grand défi d'Anjou Recherche est de franchir le pas entre l'idée née dans les laboratoires et la première réalisation sur le terrain. Cette démarche est possible au sein du Groupe Générale des Eaux grâce à l'étroite collaboration entre la recherche, l'ingénierie (avec les constructeurs OTV ou les équipementiers Trailigaz) et l'exploitation (avec les centres régionaux ou les filiales).

Les récents progrès en traitement de l'eau potable reflètent la philosophie du travail: allier des technologies connues pour constituer un nouvel ensemble plus performant. La décantation ultra-rapide Actiflo, qui rassemble les principes déjà connus de flocculation à floc lesté par ajout de microsable avec la clarification lamellaire, illustre cette démarche. Le chemin du premier pilote à MaisonsLaffitte jusqu'à la nouvelle tranche de Neuilly-sur-Marne $\left(200000 \mathrm{~m}^{3} / \mathrm{j}\right)$ s'est fait en cinq ans (1).

En se basant sur des méthodes éprouvées puis modifiées d'une façon innovante, les risques sont réduits et le développement est accéléré. Un autre exemple en traitement d'eau de barrage est l'ozoflottation. Le principe bien connu de séparation par microbulles est associé aux bulles d'ozone, ce qui a un effet de coagulation sur les algues. Là encore, la réalisation de l'usine de La Pape près de Lyon $\left(150000 \mathrm{~m}^{3} / \mathrm{j}(2)\right)$ s'est accomplie en moins de cinq ans après la première mise en œuvre sur pilote. Les premiers pilotes " Actiflo » et « Ozoflot » tournent aujourd'hui chez nos voisins européens (3). 


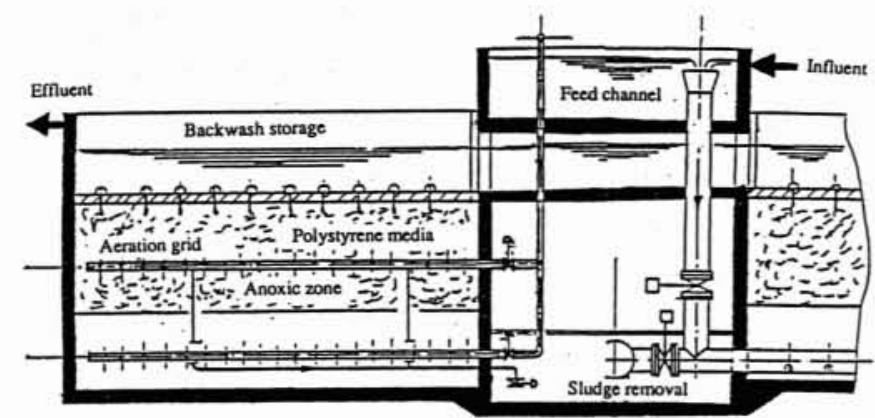

1. Vue schématique du Biostyr-Biofiltre flottant ascendant.

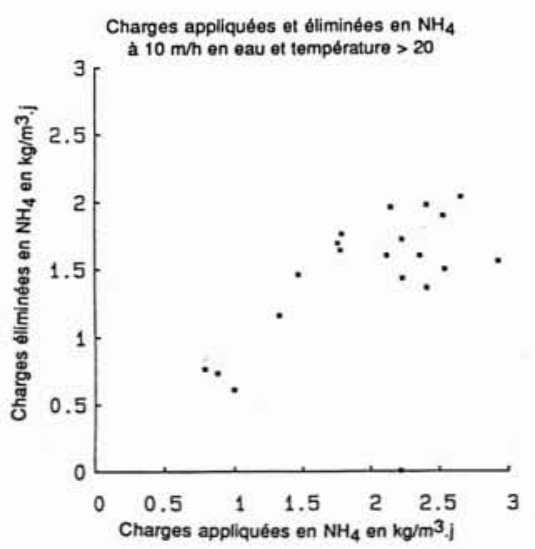

2. Nitrification sur le Biostyr de Colombes.

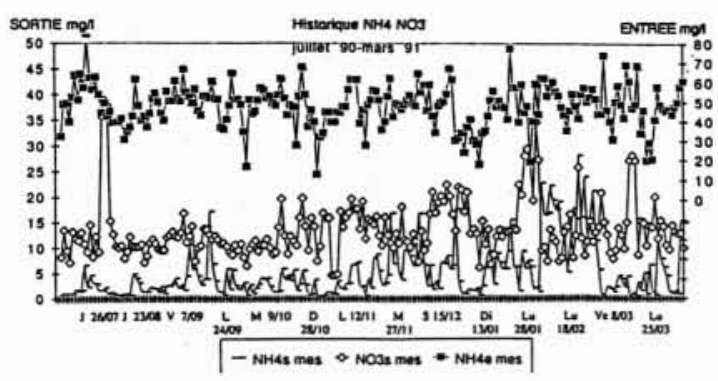

3. Résultats du Biostyr à Rambouillet.

Vitesse d'alimentation $1,5 \mathrm{~m} / \mathrm{h}$

Recirculation $200 \%$.

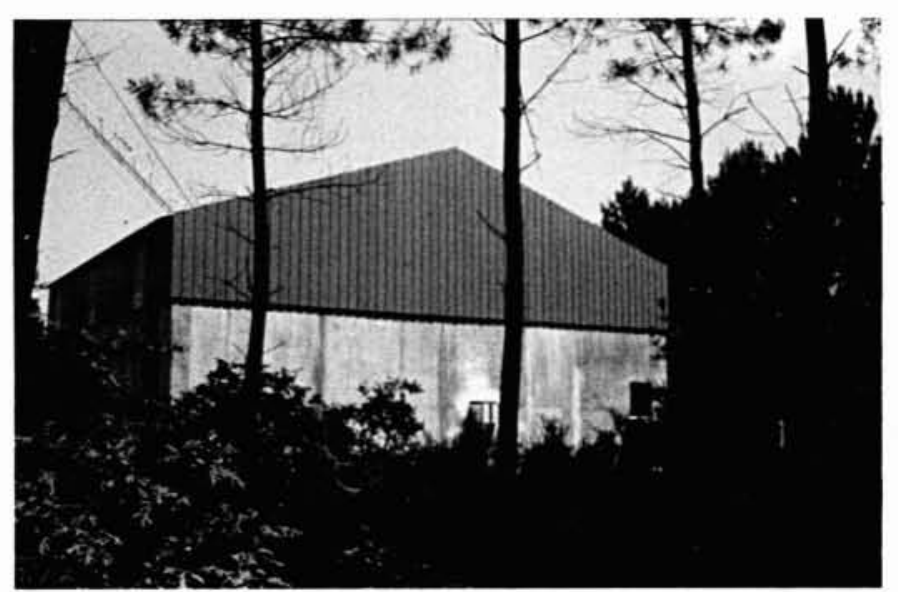

4. La station d'épuration de St-Jean-d'Illac.

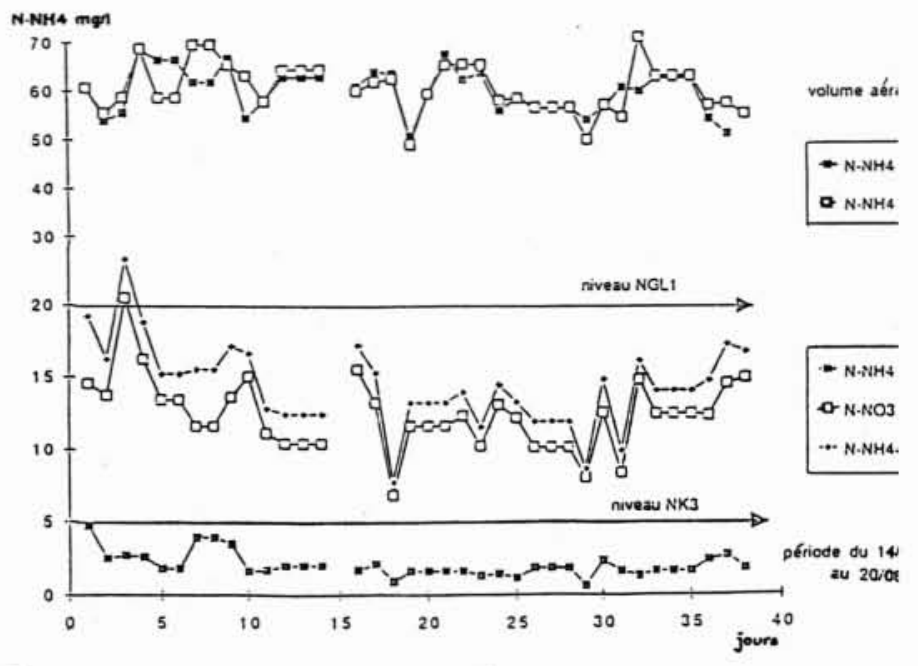

5. Concentration en azote dans l'influent et l'effluent de St-Jeand'Illac.

$\mathrm{EB}=$ eau brute

$\mathrm{ED}=$ eau décantée.

$\mathrm{ES}=$ sortie.

\section{Biofiltration aérée : la révolution a dix ans}

En épuration des eaux usées, domaine ou l'évolution technique a été particulièrement lente, un vent innovent souffle également. Pour compléter le procédé le plus appliqué en traitement biologique, les boues activées, dont les brevets datent d'avant la première guerre mondiale, OTV avait lancé il y a dix ans le Biocarbone. Cette technologie associe la fixation des bactéries, utilisée depuis des décennies dans des lits bactériens, avec la filtration des effluents, principe de base en eau potable.
Ainsi, dans un seul ouvrage se combinent dégradation des polluants solubles et rétention des matières en suspension, en augmentant en plus la concentration des bactéries. Résultat: depuis la première réalisation à Soissons en 1982, la compacité du procédé a engendré toute une nouvelle génération de stations d'épuration (4).

Implantées dans les zones urbaines, à proximité des habitants à l'origine des rejets, ces stations invisibles réduisent les coûts des infrastructures et s'intègrent dans l'environnement grâce à la parfaite maîtrise des nuisances : ne rien voir, ne rien entendre, ne rien sentir. Les visiteurs 
" éconscients " cherchent en vain les stations d'épuration le long de la côte d'Azur (Monaco, Antibes, Sanary Bandol, St-Tropez, La Ciotat, Toulon...) ou dans la région des Jeux Olympiques (Aime-La Plagne, Bourg-St-Maurice, Passy), non pas parce que ces lieux sensibles ne sont pas équipés de stations de traitement, mais parce que les ouvrages sont parfaitement dissimulés dans le paysage (5).

Cependant une nouvelle contrainte pour le traitement des eaux usées fait son apparition : l'eutrophisation croissante des cours d'eau intérieurs a déclenché une réglementation européenne très sévère sur l'azote et le phosphore. Dès 1986, le Centre de Recherche de Maisons-Laffitte se lance, avec le soutien de l'Agence de Bassin SeineNormandie, dans un programme de recherche pour développer de nouveaux procédés de traitement de l'azote.

En effet, les nouvelles normes européennes nécessitent une multiplication par quatre environ des ouvrages de traitement par des techniques classiques pour aboutir à un volume de traitement d'environ 200 litres par personne, soit l'équivalent d'une baignoire par habitant. Ce qui nécessite $0,1 \mathrm{~m}^{2}$ ou un siège de stade par personne, soit beaucoup d'espace si on pense à tous les stades qu'il faudrait construire en France pour épurer les effluents de 100 millions de personnes (en incluant les équivalents industriels). Il est également possible d'intégrer la station de dépollution sous le stade, comme à Marseille, mais c'est une autre histoire (6).

Nous sommes en 1986. Questions : comment faire proliférer les bactéries nitrifiantes qui oxident l'ammoniaque en nitrates mais qui se multiplient très lentement ? Comment intégrer l'avance technologique sur les bactéries fixées en élimination de l'azote (Biocarbonate, Biodénit - $(7,8))$ ? Comment simplifier et améliorer ces procédés? Réponse : en regardant le monde à l'envers. $\mathrm{Si}$ au lieu d'utiliser un filtre classique sur matériau lourd on prenait des billes flottantes? Et voilà lancé le premier petit réacteur, de $20 \mathrm{~cm}$ de diamètre, rempli de polystyrène, pour un premier test (9).

Essai concluant. Après quelques semaines, les bactéries s'accrochent et battent tous les records d'efficacité. Il fallait donc imaginer un biofiltre renversé, en allant à l'encontre du raisonnement habituel (fig. 1), puis chercher des sites d'application, et enfin des décideurs qui se laisseraient convaincre par l'installation d'un procédé révolutionnaire chez eux. Les deux prototypes industriels sont décrits dans les paragraphes suivants.

\section{Polystyrène blanc et pour la paix de l'esprit : le Biostyr de Colombes}

Le Syndicat Interdépartemental pour l'Assainissement de l'Agglomération Parisienne (SIAAP) avec son CRITER (Centre de Recherche Interdépartemental pour le Traitement des Eaux Résiduaires) cherche désespérément une technique pour mettre à niveau Achères $(250 \mathrm{ha}$, la plus grande station d'épuration d'Europe), sans devoir la multiplier par quatre - qui accepterait de raser la Forêt de St- Germain pour la recouvrir de bassins d'aération?
La figure 2 montre les résultats obtenus sur le pilote de Colombes, une cellule de filtration de $7 \mathrm{~m}^{2}$. En période estivale, les charges éliminées en azote dépassent $1,5 \mathrm{~kg} / \mathrm{m}^{2} . \mathrm{j}$. On pourrait donc alimenter les filtres à $10 \mathrm{~m} / \mathrm{h}$ avec un effluent clarifié de $20 \mathrm{mg} \mathrm{N}-\mathrm{NH}_{4} / 1$ sur un lit de $3 \mathrm{~m}$. On arrive à une nitrification en 20 minutes, alors que les stations de boues activées nécessitent un temps de séjour minimum de l'ordre de 10 heures. Un premier prototype à l'échelle d'Achères, quinze fois plus grand que le premier réacteur, sera démarré sur site fin 1992.

\section{Le sommet technique à Rambouillet}

A $50 \mathrm{~km}$ de Paris, une ville connue pour son environnement paisible, domaine de chasse du Président de la République, nécessitait de manière urgente une réhabilitation de sa station d'épuration pour sauver le ruisseau qui y prend sa source, la Guéville. Grâce à l'action concertée de l'Agence de l'Eau Seine-Normandie, le Syndicat des Eaux de la Région et son fermier, la Compagnie des Eaux de Rambouillet, ainsi que l'exploitant de la station d'épuration, OTV, une expérience unique est tentée avec le procédé Biostyr.

L'installation de démonstration de Rambouillet est constituée par une cellule de $22 \mathrm{~m}^{2}$. Cette station de 40000 équivalent habitants doit être mise à niveau pour l'azote et le phosphore. Avant d'entamer la construction d'une batterie de 10 filtres Biostyr, les performances devaient être vérifiées. La figure 3 montre les résultats obtenus sur un lit filtrant de $3 \mathrm{~m}$ dont le tiers inférieur était maintenu en anoxie, combinant ainsi dans un ouvrage toute l'épuration biologique jusqu'au niveau le plus exigeant.

La vitesse d'alimentation variait entre 1 et $2 \mathrm{~m} / \mathrm{h}$, ce qui se traduisait par un temps de séjour de $2 \mathrm{~h}$ dans l'ouvrage biologique. La DCO d'entrée de l'eau décantée était en moyenne de $450 \mathrm{mg} / \mathrm{l}$ et le rapport $\mathrm{DCO} / \mathrm{N}-\mathrm{NH}_{4}$ de 10 . $\mathrm{La}$ qualité de l'effluent dépendait des conditions d'aération, de température et de charge, mais se situait en moyenne autour des $15 \mathrm{mg} / \mathrm{l}$ exigés par la norme européenne. Les performances atteintes se sont avérées supérieures à celles des stations conventionnelles puisqu'obtenues dix fois plus vite.

Après une bonne année d'essais, démarrés fin 1988, les impressions sont plutôt favorables : on aboutit effectivement à un rejet conforme aux normes les plus sévères en deux heures de temps, contre une journée auparavant. Cette expérience positive encourage l'application du procédé à d'autres sites.

La première mondiale est tentée par un Syndicat girondin, mené par son président courageux : la station d'épuration de St-Jean-d'Illac est inaugurée début 1991 pour presque 15000 équivalent-habitants (fig. 4). Depuis, cette station a pleinement confirmé le potentiel du Biostyr. La figure 5 montre les résultats obtenus pendant la période de démonstration avec suivi intensif par les autorités. 


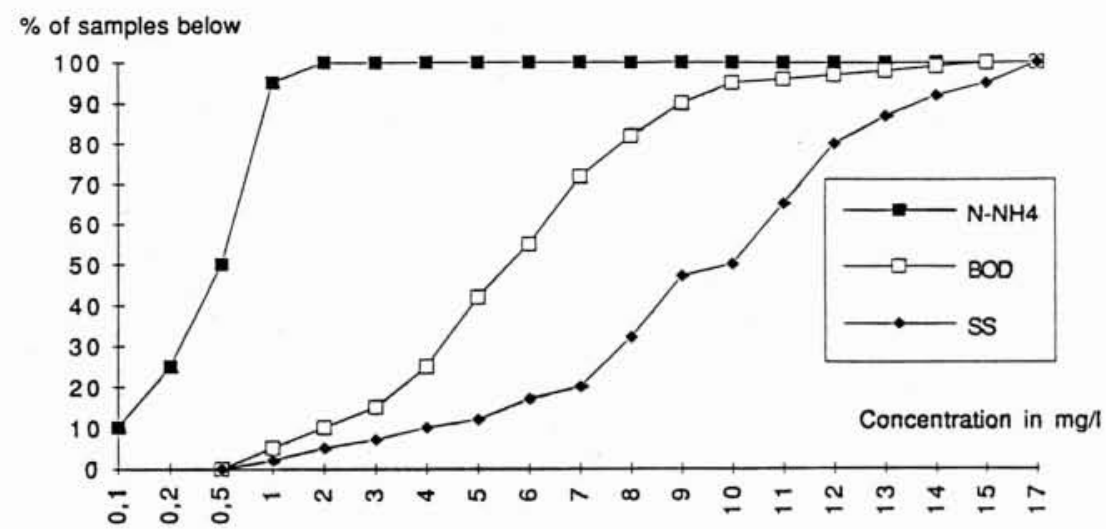

6. Pilote Biostyr tertiaire à Davyhulme. Distribution de la qualité de l'effluent.

Table 1. Références du procédé Biostyr

\begin{tabular}{|c|c|c|c|c|}
\hline & Size (m2) & Flow $\mathrm{m} 3 / \mathrm{d}$ & Startup Year & Treatment Objective \\
\hline \multicolumn{5}{|l|}{ In Operation } \\
\hline Colombes & 7 & 1000 & 1988 & Tertiary Nitrification \\
\hline Rambouillet & 22 & 2500 & 1988 & Secondary Nitrogen Removal \\
\hline St Jean d'Tllac & $5 \times 16$ & 2000 & 1990 & $\mathrm{TN}<20 \mathrm{mg} / 1$ \\
\hline \multicolumn{5}{|l|}{ Under Construction } \\
\hline Cergy & $15 \times 63$ & 40000 & 1992 & Nitrification after A-stage $\mathrm{TK} N<10 \mathrm{mg} / 1$ \\
\hline Nyborg (DK) & $8 \times 63$ & 15000 & 1992 & $\mathrm{TN}<8 \mathrm{mg} / 1 \mathrm{TP}<1,5 \mathrm{mg} / 1$ \\
\hline Evreux & $4 \times 63$ & 20000 & 1992 & Tertiary Nitrification $\mathrm{TKN}<10 \mathrm{mg} / 1$ \\
\hline Achères & 110 & 15000 & 1992 & Tertiary Nitrification \\
\hline Davyhulme (Manchester) & 63 & 7500 & 1992 & Tertiary Nitrification N-NH4 $<4 \mathrm{mg} / 1$ \\
\hline Zaragoza (Spain) & 63 & 7500 & 1992 & Tertiary Nitrification $\mathrm{N}-\mathrm{NH} 4<4 \mathrm{mg} / 1$ \\
\hline \multicolumn{5}{|l|}{ Ordered Plants } \\
\hline Manchester & $40 \times 110$ & 500000 & 1995 & Tertiary Nitrification N-NH4 $<4 \mathrm{mg} / 1$ \\
\hline Frederikshavn (DK) & $8 \times 63$ & 15000 & 1994 & $\mathrm{TN}<8 \mathrm{mg} / 1 \mathrm{TP}<1,5 \mathrm{mg} / 1$ \\
\hline Roma & $16 \times 110$ & 200000 & 1994 & $\mathrm{TN}<10 \mathrm{mg} / 1$ \\
\hline
\end{tabular}

\section{L'évolution écologique et l'expansion européenne}

L'environnement ne connaît plus de frontières et représente un véritable marché pour l'Europe. Un partenaire danois d'OTV, Envitec, essaie de répondre au programme ambitieux du plan de l'environnement pour la Mer Baltique par de nouvelles technologies. Il y a plusieurs usines existantes à mettre à niveau dans des sites restreints. L'alternative est la suivante : gagner du terrain sur la mer ou essayer des techniques plus compactes. Après comparaison des coûts, deux villes danoises se lancent dans des essais pilotes de biofiltres.

Convaincue par les performances, la ville de Nyborg signe début 1991 avec Envitec/OTV un contrat pour une station de $60000 \mathrm{~m}^{3} / \mathrm{j}$ répondant aux normes les plus stric- tes d'Europe. De plus, le réacteur biologique ne prendra pas plus de place que le décanteur primaire existant. La ville de Frederikshaven suit le même chemin : mise à niveau d'un traitement pour 100000 habitants avec le Biostyr.

Les grandes sociétés d'eau anglaises, dans leur nouveau dynamisme de privatisées, s'intéressent également aux procédés innovants pour réduire les coûts des grands travaux de réhabilitation. C'est en Grande-Bretagne qu'avait commencé l'épuration urbaine au début du siècle. Une analyse de la plus ancienne usine de boues activées du monde, Davyhulme à Manchester, montre que les Biostyr épargneraient environ un tiers de l'investissement comparé aux techniques classiques. C'est ainsi qu'un prototype de démonstration est implanté sur le site même où avait été inventé le procédé à boues activées en 1914. Les premiers 


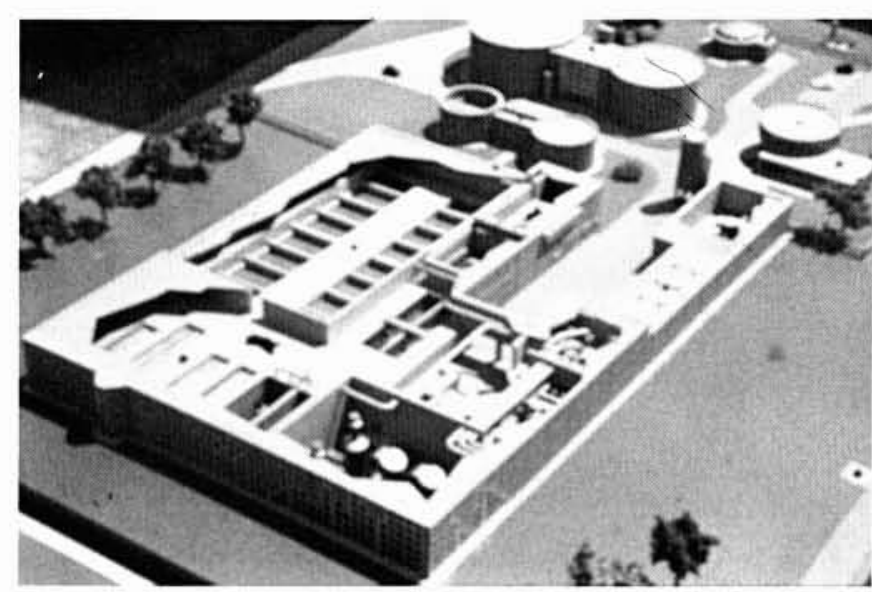

7. La station de Cergy.

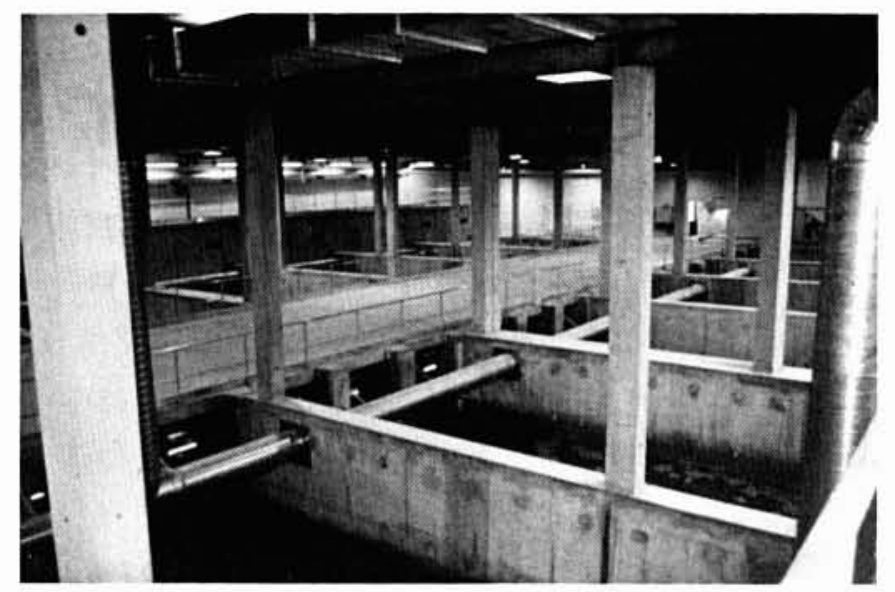

8. Filtres Biostyr à Cergy. résultats pilotes, illustrés en figure 6, confirment qu'il est possible de respecter les normes imposées sur $95 \%$ d'échantillons instantanés. Ces essais concluants déboucheront sur une usine épurant les eaux usées de plus d'un million d'habitants.

Que se passe-t-il en France ? Le défi de réaliser la station de l'an 2000 dès maintenant est relevé. Pour la construction de la station de Cergy en Ile-de-France, le Biostyr fait partie intégrante d'une usine compacte de 200000 équi- valent habitants sur $7000 \mathrm{~m}^{2}$, démarrage prévu au printemps 1992. La figure 7 montre une vue de la station, et la figure 8 les filtres Biostyr de Cergy. Cette réalisation sera fidèle à la nouvelle conception écologique des stations d'épuration : couverture des ouvrages et désodorisation un bon voisin sans nuisances. Le tableau 1 résume l'ensemble des usines Biostyr en construction. Les essais récents se concentrent sur l'adaptation du procédé à la biodéphosphatation.

\section{Conclusion}

L'adaptation d'un nouveau procédé d'épuration aux contraintes d'exploitation industrielle et aux exigences des normes européennes a été démontrée. Le procédé de biofiltration Biostyr permet d'éliminer l'azote des eaux usées, donc d'aller plus loin que les procédés conventionnels, tout en prenant moins de place. Cette compacité facilite l'intégration des ouvrages de traitement dans les agglomérations en les rapprochant des sources de pollution. Ainsi, les stations d'épuration de demain ne formeront plus des plaies dans le paysage loin des villes, mais seront des centres de dépollution écologiques, dissimulés dans l'environnement et dont les habitants seront fiers. 


\section{Bibliographie}

[1] J.-C. Dernaucourt, F. De Dianous, J.-C. Druoton, A. Grimaud, 1990. - Un nouveau procédé de clarification à très haute compacité. L'eau, l'industrie, les nuisances, mars, $\mathrm{n}^{\circ} 135$.

[2] M. Faivre, M. Delaye, J.-C. Tournier, J.N. Butte, 1991. - Starting of the La Pape water treatment plant, Urban Community of Lyon. I.O.A. 10th Ozone World Congress and Exhibition, March 19-21, Monaco.

[3] M.-M. Bourbigot, N. Martin, M. Faivre, K. Le Corre, S. QUENNELL, 1991. - Efficiency of an ozoflotation-filtration process for the treatment of the River Thames at Walton Works. J. Water SRT, Aqua, vol. 40, n² 2, pp. 88-96.

[4] J. Siвony, 1983. - Application industrielle des bactéries fixées en matière d'épuration des eaux résiduaires. $5^{\text {es }}$ Journées Scientifiques et Techniques, octobre, Lille.

[5] F. Rogalla, G. Roudon, J. Sibony, F. Blondeau, 1986. Minimising nuisances by covering compact sewage treatment plants. Wat. Sci. Tech., vol. 25, n $^{\circ} 4 / 5$, pp. 363-374.

[6] R. Thepot, G. Lavergne, 1987. - La station d'épuration de Marseille : traitement des eaux usées. T.S.M., septembre, pp. 361-373.

[7] P. Sauvegrain, F. Rogalla, M. Payraudeau, J. Sibony, 1990. - Reduced hydraulic detention time for complete nutrient removal with innovative biological reactors. Aquatech 90, septembre, Amsterdam.

[8] P. Ravarini, F. Rogalla, G. De larminat, J. Coutelle, 1990. - Large scale biological nitrate and ammonia cremoval. J. IWEM, vol. 4, August, pp. 319-329.

[9] F. Rogalla, M.-M. Bourbigot, 1990. - New developments in complete nitrogen removal with biological aerated filters. Wat. Sci. Tech., vol. 22, n ${ }^{\circ}$ 1/2, pp. 273-280.

[10] F. Rogalla, M. Badard, F. Hansen, P. Dansholm, 1992. - Upscaling a compact nitrogen removal process. Wat. Sci. Tech., vol. 26, n 5-6, pp. 1067-1076.

[11] F. Rogalla, J. Sibony, B. Lacamp, F. Hansen, 1991. Aerated biofilters : Recent European examples. IWEM Scientific Section Symposium in Advanced Sewage Treatment, 26 November 1991, London.

[12] R. Franci-Gonçalves, F. Rogalla, 1992. - Continuous biological phosphorus removal in a biofilm reactor. Wat. Sci. Tech., vol. 26, n 9-11, pp. 2027-2030. 